\title{
THE EFFECT OF CONTROL ENVIRONMENT, RISK ASSESSMENT, CONTROL ACTIVITIES, INFORMATION AND COMMUNICATION, AND MONITORING TOWARD FRAUD PREVENTION IN THE LOCAL GOVERNMENT OF PALU
}

\author{
Fattah Vitayanti*, Astriyani Nini \\ University of Tadulako, Palu, Indonesia \\ ${ }^{*}$ E-mail: webadmin@untad.ac.id
}

\begin{abstract}
This study has several objectives. The first is to examine the overall effect of Control Environment, Risk Assessment, Control Activities, Information and Communication, and Monitoring toward fraud prevention in the local government of Palu. The second is to examine the partial effect of Control Environment, Risk Assessment, Control Activities, Information and Communication, and Monitoring toward fraud prevention in the local government of Palu. The result of statistical test using multiple regression analysis with the help of SPSS 16.0 program shows these followings. First, Control Environment, Risk Assessment, Control Activities, Information and Communication, and Monitoring show overall significant effect toward fraud prevention in the local government of Palu. Second, Control Environment has no significant effect toward fraud prevention in the local government of Palu. Third, Risk Assessment has significant effect toward fraud prevention in the local government of Palu. Fourth, Control Activities have no significant effect toward fraud prevention in the local government of Palu. Fifth, Information and Communication has no significant effect toward fraud prevention in the local government of Palu. Sixth, Monitoring has significant effect toward fraud prevention in the local government of Palu.
\end{abstract}

\section{KEY WORDS}

Control environment, information, communication, fraud, local government.

Management of local government budget has not provided an overview of transparent, independent, accountable, and fair management to achieve financial performance in accordance with the vision and mission of the government. This is due to the inadequacy of organizational instruments to create good management and no high commitment from budget managers. As a result, there are various irregularities, misappropriation, and corruption. Fraud becomes something commonplace in government agencies.

Fraud is a crime of information manipulation with the aim of making the most profit ever. Usually the crime committed is to manipulate financial information. Fraud can happen anywhere, even in organizations that already have a good control system. There is no guarantee that such organization is free from the possibility of Fraud. Fraud can bring financial loss, reputation damage, legal problems, until the bankruptcy of the company.

The government has adopted a program to eliminate fraud. This is indicated by the increasing role of the Corruption Eradication Commission (KPK), the prosecution, the police, or the Court for Corruption Crimes (Tipikor Court); yet, this has not turned into a commitment to be consistently run. Fraud can inflict an unimaginable catastrophe regardless of size or type of the organization; it can happen in all places and levels, from the administrative level to the leadership level, and thus all government organizations can become victims of fraud.

Gary W. Adams et al. (in Purba, 2015) defines fraud as "The use of a person's position to enrich himself or herself through intentional misuse or deviation from the resources or assets of the company or organization".

Another definition given by three of the world's leading auditing organizations (IIA, AICPA, and ACFE1) emphasizes that fraud victims can be individuals, not just corporations or organizations. Managing the Business Risk of Fraud: a Practical Guide (2010), the three organizations define fraud as "any deliberate or delinquent action planned to deceive others to the detriment of the victim and / or benefit the perpetrator." 
Based on inspectorate examination on all SKPD (Government agencies) in Palu, from the last 5 years data, many number of losses incurred, which certainly gives negative impact to the management of local finance. Data on the loss of the government of Palu from 2010 to 2014, in 2013 the amount of losses was IDR 280,338,572 and the total liability of deposits amounting to IDR 40,791,759, which had to be IDR $321,130,331$. The data shows cases of fraud in the government agencies of Palu. The fact should awaken all parties, especially financial managers in each SKPD, to build commitment to the implementation of good financial governance consistently and widespread in all layers; without awareness and commitment, it will result in bad financial performance in government institutions.

The role of government in tackling fraud on August 28, 2008 was carried out through the renewal of organizational management in government agencies with the promulgation of Government Regulation Number 60 of 2008 on Government Internal Control System (SPIP) which is a derivative of the Law Number 1 of 2004 on State Treasury, as the act indicates the need for SPIP to be further regulated in a government regulation.

The Government Internal Control System (SPIP) comprises assessment of control environment, risk assessment, control activities, information and communication, and monitoring. SPIP is a system of internal control held comprehensively in the environment of central government and local government, in which the internal control system is an integral process on the actions and activities undertaken continuously by the leaders and all levels of employees to provide reasonable assurance on the achievement of organizational goals through effective and efficient activities, reliability of financial reporting, security of state assets, and compliance with laws and regulations.

AICPA Professional Standards, as cited by Konrath in Agoes (2012) identifies five internal control components: control environment, risk assessment, information and communication, control activities, and monitoring. The first component, the control environment, is the foundation of the other four components.

Based on the above description, the authors find it important to conduct research under the heading "The Effect of Control Environment, Risk Assessment, Control Activities, Information and Communication and Monitoring toward Fraud Prevention in the Local Government of Palu“.

The objectives of the study are as follows: to examine the overall effect of control environment, risk assessment, control activities, information and communication, and monitoring toward fraud prevention in the local government of Palu; and to examine the partial effect of control environment, risk assessment, control activities, information and communication, and monitoring toward fraud prevention in the local government of Palu.

\section{METHODS OF RESEARCH}

The method used was verificative research with quantitative approach. Verificative research is basically to test the theory by testing the hypothesis. Hypothesis testing is done by using statistical calculation, which is used to test the effect of variables. The research is done at Local Government agencies (SKPD) of Palu with the consideration that all SKPDs are authorized in managing the budget and audited every year. Research population was all agencies of local government of Palu, which become the object of inspection from the Inspectorate of Palu and are under the Organization of Administration of Palu, as many as 87 SKPDs. The research instrument was Likert scale. In Likert scale, respondents would be given a question with some alternatives considered by respondents most appropriate. The research instrument (questionnaire) must first be tested for its accuracy and reliability. The method used to analyze the data in this study is Multiple Linear Regression. The analysis requires that the data used in the calculation be at least an interval-scale data.

\section{RESULTS AND DISCUSSION}

The control environment consists of eight indicators, namely the enforcement of integrity and ethical values, commitment to competence, conducive leadership, 
organizational structure, delegation of authority and responsibility, policies and practices of human resources development, realization on the role of effective internal control officers, and good relationship with relevant government agencies.

The indicators of enforcement of integrity and ethical values based on the answers of the majority of respondents indicate that conducive management in realizing the integrity and ethical values has been implemented very well by SKPDs. This is done so the code of ethics and enforcement mechanisms are implemented in accordance with applicable provisions. Then, it can be concluded that SKPD Palu has applied the indicators in the control environment.

Risk assessment consists of five indicators, namely goal setting of agencies as a whole, goal setting at activity level, risk identification, risk analysis, and managing risk during change.

Risk assessment is used to assess the government's internal control system. The majority of respondents illustrates that in general the local government of Palu has implemented risk assessment adequately. In other words, the internal control system in Palu generally has been able to analyze, manage, and provide an assessment of the risks faced by government agencies, both from external and internal parties.

Control activities consist of the general application of policies and procedures, reviews on the performance of relevant government agencies, human resource development, control over the management of information systems, physical control over assets, stipulation and review of indicators and performance measures, segregation of functions, authorization of transactions and important events, accurate and timely recording of transactions and events, restrictions on access to resources and records, accountability to resources and records, as well as good documentation of internal control systems.

For the internal control of the government, the majority of respondents state that in general, the control activities within the Palu government have been applied adequately, effectively, and efficiently in achieving organizational goals.

Information and communication consists of information, communication, as well as forms and means of communication.

For the internal control of the government, the majority of respondents state that the government of Palu has applied the right elements of information and good communication to support the implementation of Government Internal Control System.

Monitoring consists of continuous monitoring, separate evaluation, and completion of the audit. Monitoring activities have been running well to support the implementation of Government Internal Control System.

To do fraud, the perpetrator must have the opportunity to implement and conceal the action, and the rationalization that the actions are not criminal activity. Fraud based on the responses of respondents is illustrated as follows:

The majority of respondents agreed upon the prevention of fraud on SKPD of Palu as a whole by using the afore-mentioned three indicators. The total mean was 4.18 meaning that the assessment of the overall indicators is in the category of good. This means the local government of Palu has made efforts in prevention of fraud.

Based on the results of multiple linear regression analysis using SPSS for Wind Release 16.0 software, the results of 87 SKPD (respondents) on the influence of the two independent variables (Control Environment, Risk Assessment, Control Activities, Information and Communication, and Monitoring) on fraud prevention are presented in Table 7.

The obtained regression mode from Table 7 is as follows:

$$
Y=9.723+0.183 X_{1}+0.430 X_{2}+0.030 X_{3}-0.022 X_{4}+0.928 X_{5}
$$

Simultaneously control environment, risk assessment, control activities, information and communication, and monitoring significantly influence fraud prevention in the loal government of Palu based on F-Test results. 
RJOAS, 5(65), May 2017

Table 1 - Frequency Distribution on Respondents' Answers about Control Environment

\begin{tabular}{|c|c|c|c|c|c|c|c|c|c|c|c|c|c|c|c|}
\hline \multirow{3}{*}{ No } & \multirow{3}{*}{ Items } & \multicolumn{10}{|c|}{ Alternatives } & \multirow{3}{*}{$\mathrm{N}$} & \multirow{3}{*}{\multicolumn{2}{|c|}{ Total Score }} & \multirow{3}{*}{$\begin{array}{l}\text { Average Score per } \\
\text { Item }\end{array}$} \\
\hline & & \multicolumn{2}{|r|}{5} & \multicolumn{2}{|r|}{4} & & \multicolumn{2}{|r|}{2} & \multicolumn{2}{|r|}{1} & & & & \\
\hline & & $\mathrm{F}$ & $\%$ & $\mathrm{~F}$ & $\%$ & $\mathrm{~F}$ & $\%$ & $\mathrm{~F}$ & $\%$ & $\mathrm{~F}$ & $\%$ & & & & \\
\hline \multicolumn{16}{|c|}{ Indiators on enforcement of integrity and ethical values } \\
\hline 1 & Speed in responding to problems & 33 & 37.93 & 51 & 58.62 & 2 & 2.30 & 0 & - & 1 & 1.15 & 87 & \multicolumn{2}{|c|}{376} & 4.32 \\
\hline 2 & Application of good ethics & 23 & 26.44 & 62 & 71.26 & 2 & 2.30 & 0 & - & 0 & - & 87 & \multicolumn{2}{|c|}{369} & 4.24 \\
\hline 3 & Sanctions & 40 & 45.98 & 44 & 50.57 & 2 & 2.30 & 1 & 1.15 & 0 & - & 87 & \multirow{2}{*}{\multicolumn{2}{|c|}{$\begin{array}{l}384 \\
363\end{array}$}} & 4.41 \\
\hline 4 & Realistic goal setting & 22 & 25.29 & 60 & 68.97 & 3 & 3.45 & 2 & 2.30 & 0 & & 87 & & & 4.17 \\
\hline \multicolumn{3}{|c|}{ Mean } & 33.91 & & 62.36 & & 2.59 & & 0.86 & & 0.29 & & \multicolumn{2}{|c|}{373} & 4.29 \\
\hline \multicolumn{16}{|c|}{ Indiators on commitment to competence } \\
\hline 5 & Job analysis and supervision & 31 & 35.63 & 53 & 60.92 & 1 & 1.15 & 2 & 2.30 & 0 & - & 87 & \multicolumn{2}{|c|}{374} & 4.30 \\
\hline 6 & Expertise & 35 & 40.23 & 45 & 51.72 & 4 & 4.60 & 2 & 2.30 & 1 & 1.15 & 87 & 37 & & 4.28 \\
\hline 7 & Employee training & 16 & 18.39 & 64 & 73.56 & 5 & 5.75 & 1 & 1.15 & 1 & 1.15 & 87 & 35 & & 4.07 \\
\hline 8 & Performance improvement guidance & 27 & 31.03 & 58 & 66.67 & 1 & 1.15 & 1 & 1.15 & 0 & - & 87 & 37 & & 4.28 \\
\hline & Mean & & 31.32 & & 63.22 & & 2.53 & & 1.72 & & 0.57 & & 368. & & 4.23 \\
\hline & tors on conducive leadership & & & & & & & & & & & & & & \\
\hline 9 & Consideration of risks in decision-making & 39 & 44.83 & 46 & 52.87 & 2 & 2.30 & & - & & - & 87 & 385 & 4.43 & \\
\hline 10 & Responsive to financial reporting & 45 & 51.72 & 42 & 48.28 & & - & & - & & - & 87 & 393 & 4.52 & \\
\hline 11 & Budgeting based on reasonable price & 8 & 9.20 & 37 & 42.53 & 33 & 37.93 & 5 & 5.75 & 4 & 4.60 & 87 & 301 & 3.46 & \\
\hline & Mean & & 35.25 & & 47.89 & & 13.41 & & 1.92 & & 1.53 & & 359.67 & 4.13 & \\
\hline & tors on organizational strucure & & & & & & & & & & & & & & \\
\hline 12 & Organizational structure according to the nature of activity & 17 & 19.54 & 57 & 65.52 & 11 & 12.64 & 2 & 2.30 & & - & 87 & 350 & 4.02 & \\
\hline 13 & The existence of an organization chart & 23 & 26.44 & 62 & 71.26 & 2 & 2.30 & & - & & - & 87 & 369 & 4.24 & \\
\hline 14 & Determination of the appropriate number of employees & 20 & 22.99 & 55 & 63.22 & 8 & 9.20 & 1 & 1.15 & 3 & 3.45 & 87 & 349 & 4.01 & \\
\hline & Mean & & 22.99 & & 66.67 & & 8.05 & & 1.15 & & 1.15 & & 356.00 & 4.09 & \\
\hline & ators of delegation of authority and responsibility & & & & & & & & & & & & & & \\
\hline 15 & The right authority & 36 & 41.38 & 48 & 55.17 & 2 & 2.30 & & - & 1 & 1.15 & 87 & 379 & 4.36 & \\
\hline 16 & Resolving the problem according to responsibilities & 28 & 32.18 & 55 & 63.22 & 3 & 3.45 & 1 & 1.15 & & - & 87 & 371 & 4.26 & \\
\hline 17 & Supervision of the leaders & 12 & 13.79 & 65 & 74.71 & 7 & 8.05 & 3 & 3.45 & & - & 87 & 347 & 3.99 & \\
\hline & Mean & & 29.12 & & 64.37 & & 4.60 & & 1.53 & & 0.38 & & 365.67 & 4.20 & \\
\hline & ators of policies and practices of human resources development & & & & & & & & & & & & & & \\
\hline 18 & Placement of employees according to standards and criteria & 23 & 26.44 & 58 & 66.67 & 3 & 3.45 & 2 & 2.30 & 1 & 1.15 & 87 & 361 & 4.15 & \\
\hline 19 & Sanctions on violation of the code of ethics & 32 & 36.78 & 48 & 55.17 & 6 & 6.90 & 1 & 1.15 & & - & 87 & 372 & 4.28 & \\
\hline 20 & Employee training & 15 & 17.24 & 65 & 74.71 & 5 & 5.75 & 2 & 2.30 & & - & 87 & 354 & 4.07 & \\
\hline & Mean & & 26.82 & & 65.52 & & 5.36 & & 1.92 & & 0.38 & & 362.33 & 4.16 & \\
\hline & ators of realization on the role of effective internal control officers & & & & & & & & & & & & & & \\
\hline 21 & Supervision of the government's internal control apparatus & 22 & 25.29 & 58 & 66.67 & 5 & 5.75 & 2 & 2.30 & & - & 87 & 361 & 4.15 & \\
\hline 22 & The existence of supervisory reports & 31 & 35.63 & 53 & 60.92 & 2 & 2.30 & 1 & 1.15 & & - & 87 & 375 & 4.31 & \\
\hline 23 & Mechanism of organizing tasks and functions & 30 & 34.48 & 53 & 60.92 & 3 & 3.45 & 1 & 1.15 & & - & 87 & 373 & 4.29 & \\
\hline & Mean & & 31.80 & & 62.84 & & 3.83 & & 1.53 & & - & & 369.67 & 4.25 & \\
\hline & ators of good relationship with relevant government agencies & & & & & & & & & & & & & & \\
\hline 24 & Improving the quality of governance in the implementation of tasks and functions & 31 & 35.63 & 50 & 57.47 & 3 & 3.45 & 3 & 3.45 & & - & 87 & 370 & 4.25 & \\
\hline 25 & The existence of a good working relationship & 27 & 31.03 & 55 & 63.22 & 3 & 3.26 & 2 & 2.17 & & - & 87 & 368 & 4.23 & \\
\hline & Mean & & 33.33 & & 60.34 & & 3.35 & & 2.81 & & - & & 369.00 & 4.24 & \\
\hline & Mean on the Variable of Control Activities & & 30.57 & & 61.65 & & 5.46 & & 1.68 & & 0.54 & & 365.42 & 4.20 & \\
\hline
\end{tabular}


Table 2 - Frequency Distribution on Respondents' Answers about Risk Assessment

\begin{tabular}{|c|c|c|c|c|c|c|c|c|c|c|c|c|c|c|}
\hline \multirow{3}{*}{ No } & \multirow{3}{*}{ Items } & \multicolumn{10}{|c|}{ Alternatives } & \multirow{3}{*}{$\mathrm{N}$} & \multirow{3}{*}{ Total Score } & \multirow{3}{*}{ Averag Score Per Item } \\
\hline & & \multicolumn{2}{|c|}{5} & \multicolumn{2}{|r|}{4} & \multicolumn{2}{|c|}{3} & \multicolumn{2}{|c|}{2} & \multicolumn{2}{|c|}{1} & & & \\
\hline & & $\mathrm{F}$ & $\%$ & $\mathrm{~F}$ & $\%$ & $\mathrm{~F}$ & $\%$ & $\mathrm{~F}$ & $\%$ & $\mathrm{~F}$ & $\%$ & & & \\
\hline \multicolumn{15}{|c|}{ Indicators of goal setting of agencies as a whole } \\
\hline 1 & Goal setting based on regulation & 33 & 37.93 & 49 & 56.32 & 4 & 4.60 & 0 & - & 1 & 1.15 & 87 & 374 & 4.30 \\
\hline 2 & Employees know the purpose of the agency & 35 & 40.23 & 46 & 52.87 & 6 & 6.90 & 0 & - & 0 & - & 87 & 377 & 4.33 \\
\hline 3 & Strategic plan and risk assessment according to goals & 26 & 29.89 & 58 & 66.67 & 1 & 1.15 & 2 & 2.30 & 0 & - & 87 & 369 & 4.24 \\
\hline \multicolumn{3}{|c|}{ Mean } & 36.02 & & 58.62 & & 4.21 & & 0.77 & & 0.38 & & 373.3 & 4.29 \\
\hline \multicolumn{15}{|c|}{ Indicators of goal setting at activity level } \\
\hline 4 & The purpose of the activity is always reviewed & 20 & 22.99 & 61 & 70.11 & 5 & 5.75 & 1 & 1.15 & 0 & - & 87 & 361 & 4.15 \\
\hline 5 & The purpose of each activity is not conflicting one another & 24 & 27.59 & 61 & 70.11 & 2 & 2.30 & & - & & - & 87 & 370 & 4.25 \\
\hline 6 & Leaders are involved in setting the objectives of the activity & 33 & 37.93 & 49 & 56.32 & 4 & 4.60 & 1 & 1.15 & 0 & - & 87 & 375 & 4.31 \\
\hline \multicolumn{3}{|c|}{ Mean } & 29.50 & & 65.52 & & 4.21 & & 0.77 & & - & & 368.67 & 4.24 \\
\hline \multicolumn{15}{|c|}{ Indicators of risk identification } \\
\hline 7 & Risk identification of audit results & 27 & 31.03 & 52 & 59.77 & 7 & 8.05 & 1 & 1.15 & & - & 87 & 366 & 4.21 \\
\hline 8 & Risk analysis & 20 & 22.99 & 65 & 74.71 & 2 & 2.30 & & - & & - & 87 & 366 & 4.21 \\
\hline 9 & Risk considerations & 10 & 11.49 & 52 & 59.77 & 23 & 26.44 & 2 & 2.30 & & - & 87 & 331 & 3.80 \\
\hline \multicolumn{3}{|c|}{ Mean } & 21.84 & & 64.75 & & 12.26 & & 1.15 & & - & & 354.33 & 4.07 \\
\hline \multicolumn{15}{|c|}{ Indicators of risk analysis } \\
\hline 10 & Determine the impact of risk, the existence of controls to reduce risk & 18 & 20.69 & 64 & 73.56 & 5 & 5.75 & & - & & - & 87 & 361 & 4.15 \\
\hline 11 & Carefully determine the level of risk & 29 & 33.33 & 56 & 64.37 & 2 & 2.30 & & - & & - & 87 & 375 & 4.31 \\
\hline 12 & Controls to reduce risk & 22 & 25.29 & 55 & 63.22 & 7 & 8.05 & 2 & 2.30 & 1 & 1.15 & 87 & 356 & 4.09 \\
\hline \multicolumn{3}{|c|}{ Mean } & 26.44 & & 67.05 & & 5.36 & & 0.77 & & 0.38 & & 364.00 & 4.18 \\
\hline \multicolumn{15}{|c|}{ Indicators of managing risk during change } \\
\hline 13 & Considerations and actions of the impact of risk & 16 & 18.39 & 62 & 71.26 & 7 & 8.05 & 2 & 2.30 & & - & 87 & 353 & 4.06 \\
\hline 14 & Consideration of the risk of new employee placement & 18 & 20.69 & 50 & 57.47 & 16 & 18.39 & 3 & 3.45 & & - & 87 & 344 & 3.95 \\
\hline 15 & Risk analysis of activities implemented & 13 & 14.94 & 61 & 70.11 & 9 & 10.34 & 4 & 4.60 & & - & 87 & 344 & 3.95 \\
\hline \multirow{2}{*}{\multicolumn{3}{|c|}{ Mean }} & 18.01 & & 66.28 & & 12.26 & & 3.45 & & - & & 347.00 & 3.99 \\
\hline & & & 26.36 & & 64.44 & & 7.66 & & 1.38 & & 0.15 & & 361.47 & 4.15 \\
\hline
\end{tabular}

Source: Primary Data Analysed, 2016. 
Table 3 - Frequency Distribution on Respondents' Answers about Control Activities

\begin{tabular}{|c|c|c|c|c|c|c|c|c|c|c|c|c|c|c|}
\hline \multirow{3}{*}{ No } & \multirow{3}{*}{ Items } & \multicolumn{10}{|c|}{ Alternatives } & \multirow{3}{*}{$\mathrm{N}$} & \multirow{3}{*}{ Total Score } & \multirow{3}{*}{ Average Score per Item } \\
\hline & & \multicolumn{2}{|c|}{5} & \multicolumn{2}{|l|}{4} & \multicolumn{2}{|l|}{3} & \multicolumn{2}{|l|}{2} & \multicolumn{2}{|l|}{1} & & & \\
\hline & & $\mathrm{F}$ & $\%$ & $\mathrm{~F}$ & $\%$ & $\mathrm{~F}$ & $\%$ & $\mathrm{~F}$ & $\%$ & $\mathrm{~F}$ & $\%$ & & & \\
\hline \multicolumn{15}{|c|}{ Indicators of the general application of policies and procedures } \\
\hline 1 & Identification of control activities & 14 & 16.09 & 66 & 75.86 & 3 & 3.45 & 4 & 4.60 & 0 & - & 87 & 351 & 4.03 \\
\hline 2 & Problems are handled in a timely manner & 16 & 18.39 & 61 & 70.11 & 6 & 6.90 & 4 & 4.60 & 0 & - & 87 & 350 & 4.02 \\
\hline \multirow[t]{2}{*}{3} & Evaluation of control activities & 22 & 25.29 & 59 & 67.82 & 5 & 5.75 & 1 & 1.15 & 0 & - & 87 & 363 & 4.17 \\
\hline & Mean & & 19.92 & & 71.26 & & 5.36 & 5 & 3.45 & & - & & 354.7 & 4.08 \\
\hline \multicolumn{15}{|c|}{ Indicators of reviews on the performance of relevant government agencies } \\
\hline 4 & Leaders are involved in the preparation of Renstra and Renja & 49 & 56.32 & 38 & 43.68 & & - & & - & 0 & - & 87 & 397 & 4.56 \\
\hline 5 & Review on performance reports & 33 & 37.93 & 49 & 56.32 & 4 & 4.60 & 1 & 1.15 & & - & 87 & 375 & 4.31 \\
\hline \multirow[t]{2}{*}{6} & Reconciliation and checking the accuracy of information & 26 & 29.89 & 54 & 62.07 & 5 & 5.75 & 2 & 2.30 & 0 & - & 87 & 365 & 4.20 \\
\hline & Mean & & 41.38 & & 54.02 & & 3.45 & & 1.15 & & - & & 379.00 & 4.36 \\
\hline \multicolumn{15}{|c|}{ Indicators of human resource development } \\
\hline 7 & Employee understanding of vision and mission & 34 & 39.08 & 47 & 54.02 & 6 & 6.90 & & - & & - & 87 & 358 & 4.11 \\
\hline 8 & Training and employee responsibilities & 20 & 22.99 & 52 & 59.77 & 10 & 11.49 & 4 & 4.60 & 1 & 1.15 & 87 & 347 & 3.99 \\
\hline \multirow[t]{2}{*}{9} & Continuous supervision & 29 & 33.33 & 53 & 60.92 & 1 & 1.15 & 4 & 4.60 & & - & 87 & 368 & 4.23 \\
\hline & Mean & & 31.80 & & 58.24 & & 6.51 & & 3.07 & & 0.38 & & 357.67 & 4.11 \\
\hline \multicolumn{15}{|c|}{ Indicators of control over the management of information systems } \\
\hline 10 & Control over information systems & 23 & 26.44 & 61 & 70.11 & 2 & 2.30 & 1 & 1.15 & & - & 87 & 367 & 4.22 \\
\hline 11 & Monitoring access to information systems & 20 & 22.99 & 58 & 66.67 & 6 & 6.90 & 3 & 3.45 & & - & 87 & 356 & 4.09 \\
\hline \multirow[t]{2}{*}{12} & Data improvement and reporting & 32 & 36.78 & 48 & 55.17 & 3 & 3.45 & 4 & 4.60 & & - & 87 & 369 & 4.24 \\
\hline & Mean & & 28.74 & & 63.98 & & 4.21 & & 3.07 & & - & & 364.00 & 4.18 \\
\hline Indic & tors of physical control over assets & & & & & & & & & & & & & \\
\hline 13 & Policies and procedures of physical security & 21 & 24.14 & 58 & 66.67 & 6 & 6.90 & 2 & 2.30 & & - & 87 & 359 & 4.13 \\
\hline 14 & Assets checked and compared to control records & 33 & 37.93 & 54 & 62.07 & & - & & - & & - & 87 & 381 & 4.38 \\
\hline 15 & Equipment kept in a safe place & 29 & 33.33 & 57 & 65.52 & 1 & 1.15 & & - & & - & 87 & 376 & 4.32 \\
\hline & Mean & & 31.80 & & 64.75 & & 2.68 & & 0.77 & & - & & 372.00 & 4.28 \\
\hline Indi & tors of stipulation and review of indicators and performance measures & & & & & & & & & & & & & \\
\hline 16 & Size and performance indicators are set in government agencies & 27 & 31.03 & 51 & 58.62 & 7 & 8.05 & 2 & 2.30 & & - & 87 & 364 & 4.18 \\
\hline 17 & Evaluation of performance appraisal measurement & 27 & 31.03 & 57 & 65.52 & 3 & 3.45 & & - & & - & 87 & 372 & 4.28 \\
\hline 18 & Performance data is analyzed and compared & 20 & 22.99 & 61 & 70.11 & 5 & 5.43 & 1 & 1.15 & & - & 87 & 361 & 4.15 \\
\hline & Mean & & 28.35 & & 64.75 & & 5.64 & & 1.15 & & - & & 365.67 & 4.20 \\
\hline Indic & tors of segregation of functions & & & & & & & & & & & & & \\
\hline 19 & All major aspects of the transaction are not controlled by one person & 22 & 25.29 & 50 & 57.47 & 11 & 12.64 & 4 & 4.60 & & - & 87 & 351 & 4.03 \\
\hline 20 & The bank balance is reconciled by the treasurer only & 15 & 17.24 & 33 & 37.93 & 21 & 24.14 & 13 & 14.94 & 5 & 5.75 & 87 & 301 & 3.46 \\
\hline 21 & Leaders reduce opportunities for collusion & 22 & 25.29 & 50 & 57.47 & 11 & 12.64 & 2 & 2.30 & 2 & 2.30 & 87 & 349 & 4.01 \\
\hline & Mean & & 22.61 & & 50.96 & & 16.48 & & 7.28 & & 2.68 & & 333.67 & 3.84 \\
\hline Indi & tors of authorization of transactions and important events & & & & & & & & & & & & & \\
\hline 22 & Only valid transactions and events can be processed & 21 & 24.14 & 54 & 62.07 & 7 & 8.05 & 5 & 5.75 & & - & 87 & 352 & 4.05 \\
\hline 23 & Authorization requirements are in accordance with legislation & 18 & 20.69 & 62 & 71.26 & 6 & 6.90 & 1 & 1.15 & & - & 87 & 358 & 4.11 \\
\hline & Mean & & 22.41 & & 66.67 & & 7.47 & & 3.45 & & - & & 355.00 & 4.08 \\
\hline Indic & tors of accurate and timely recording of transactions and events & & & & & & & & & & & & & \\
\hline 24 & Transactions are classified appropriately & 29 & 33.33 & 54 & 62.07 & 2 & 2.30 & 2 & 2.30 & & - & 87 & 371 & 4.26 \\
\hline 25 & Proper classification and recording & 20 & 21.74 & 62 & 71.26 & 4 & 4.60 & & - & 1 & 1.15 & 87 & 361 & 4.15 \\
\hline & Mean & & 27.54 & & 66.67 & & 3.45 & & 1.15 & & 0.57 & & 366.00 & 4.21 \\
\hline
\end{tabular}




\begin{tabular}{|c|c|c|c|c|c|c|c|c|c|c|c|c|c|}
\hline \multicolumn{14}{|c|}{ inue of Table 3} \\
\hline \multicolumn{14}{|c|}{ Indicators of restrictions on access to resources and records } \\
\hline 26 & Restrictions on access to resources and records & 8 & 9.20 & 56 & 64.37 & 15 & 17.24 & 8 & 9.20 & - & 87 & 325 & 3.74 \\
\hline 27 & Access restrictions for storage are reviewed and maintained & 12 & 13.79 & 63 & 72.41 & 9 & 10.34 & 3 & 3.45 & - & 87 & 345 & 3.97 \\
\hline \multirow[t]{2}{*}{28} & Consideration of appropriate access restriction level & 11 & 12.64 & 62 & 71.26 & 12 & 13.79 & 2 & 2.30 & - & 87 & 343 & 3.94 \\
\hline & Mean & & 11.88 & & 69.35 & & 13.79 & & 4.98 & - & & 337.67 & 3.88 \\
\hline \multicolumn{14}{|c|}{ Indicators of accountability to resources and records } \\
\hline 29 & Establishment of access liability & 18 & 20.69 & 64 & 73.56 & 5 & 5.75 & & - & - & 87 & 361 & 4.15 \\
\hline 30 & Comparison of resources with accountability recording & 16 & 18.39 & 68 & 78.16 & 3 & 3.45 & & - & - & 87 & 361 & 4.15 \\
\hline \multirow{2}{*}{31} & Responsibility for resource accountability & 24 & 27.59 & 61 & 70.11 & 2 & 2.30 & & - & - & 87 & 370 & 4.25 \\
\hline & Mean & & 22.22 & & 73.95 & & 3.83 & & - & - & & 364.00 & 4.18 \\
\hline \multicolumn{14}{|c|}{ Indicators of good documentation of internal control systems } \\
\hline 32 & The existence of documentation of internal control system & 17 & 19.54 & 69 & 79.31 & 1 & 1.15 & & - & - & 87 & 364 & 4.18 \\
\hline 33 & The existence of complete and accurate documentation & 25 & 28.74 & 61 & 70.11 & 1 & 1.15 & & - & - & 87 & 372 & 4.28 \\
\hline \multirow[t]{2}{*}{34} & All documents are well maintained & 30 & 34.48 & 56 & 64.37 & 1 & 1.15 & & - & - & 87 & 377 & 4.33 \\
\hline & Mean & & 27.59 & & 71.26 & & 1.15 & & 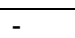 & - & & 371.00 & 4.26 \\
\hline \multicolumn{3}{|c|}{ Mean on the Variable of Control Activities } & 26.35 & & 64.66 & & 6.17 & & 2.46 & 0.30 & & 360.03 & 4.14 \\
\hline
\end{tabular}

Source: Primary Data Analysed, 2016.

Table 4 - Frequency Distribution on Respondents' Answers about Information and Communication

\begin{tabular}{|c|c|c|c|c|c|c|c|c|c|c|c|c|c|c|}
\hline \multirow{3}{*}{ No } & \multirow{3}{*}{ Items } & \multicolumn{10}{|c|}{ Alternatves } & \multirow{3}{*}{$\mathrm{N}$} & \multirow{3}{*}{ Total Score } & \multirow{3}{*}{ Average Score Per Item } \\
\hline & & \multicolumn{2}{|c|}{5} & \multicolumn{2}{|c|}{4} & \multicolumn{2}{|c|}{3} & \multicolumn{2}{|c|}{2} & \multicolumn{2}{|c|}{1} & & & \\
\hline & & $\mathrm{F}$ & $\%$ & $\mathrm{~F}$ & $\%$ & $\mathrm{~F}$ & $\%$ & $\mathrm{~F}$ & $\%$ & $\mathrm{~F}$ & $\%$ & & & \\
\hline \multicolumn{15}{|c|}{ Indicators of information } \\
\hline 1 & The presence of internal and external information & 25 & 28.74 & 55 & 63.22 & 7 & 8.05 & 0 & - & 0 & - & 87 & 366 & 4.21 \\
\hline 2 & Timely information & 21 & 24.14 & 60 & 68.97 & 4 & 4.60 & 2 & 2.30 & 0 & - & 87 & 361 & 4.15 \\
\hline 3 & Adequate financial and budget information & 26 & 29.89 & 56 & 64.37 & 5 & 5.75 & 0 & - & 0 & - & 87 & 369 & 4.24 \\
\hline \multicolumn{3}{|c|}{ Mean } & 27.59 & & 65.52 & & 6.13 & & 0.77 & & - & & 365.3 & 4.20 \\
\hline \multicolumn{15}{|c|}{ Indicators of communication } \\
\hline 4 & The establishment of effective internal communication & 39 & 44.83 & 46 & 52.87 & 2 & 2.30 & & - & 0 & - & 87 & 385 & 4.43 \\
\hline 5 & Information has been submitted to all divisions & 23 & 26.44 & 59 & 67.82 & 3 & 3.45 & 2 & 2.30 & & - & 87 & 364 & 4.18 \\
\hline 6 & Suggestions are considered and acted upon & 21 & 24.14 & 62 & 71.26 & 1 & 1.15 & 3 & 3.45 & 0 & - & 87 & 362 & 4.16 \\
\hline \multicolumn{3}{|c|}{ Mean } & 31.80 & & 63.98 & & 2.30 & & 1.92 & & - & & 370.33 & 4.26 \\
\hline \multicolumn{15}{|c|}{ Indicators on the forms and means of communication } \\
\hline 7 & The forms and means of communication are effective & 17 & 19.54 & 64 & 73.56 & 3 & 3.45 & 3 & 3.45 & & - & 87 & 356 & 4.09 \\
\hline 8 & Identifying the development of information needs & 14 & 16.09 & 67 & 77.01 & 4 & 4.60 & 2 & 2.30 & & - & 87 & 354 & 4.07 \\
\hline 9 & Leaders always monitor the quality of information & 19 & 21.84 & 61 & 70.11 & 3 & 3.45 & 4 & 4.60 & & - & 87 & 356 & 4.09 \\
\hline \multirow{2}{*}{\multicolumn{3}{|c|}{$\begin{array}{c}\text { Mean } \\
\text { Mean on the Variable of Control Activities }\end{array}$}} & 19.16 & & 73.56 & & 3.83 & & 3.45 & & - & & 355.33 & 4.08 \\
\hline & & & 26.18 & & 67.69 & & 4.09 & & 2.04 & & 0.00 & & 363.67 & 4.18 \\
\hline
\end{tabular}

Source: Primary Data Analysed, 2016. 
Table 5 - Frequency Distribution on Respondents' Answers about Monitoring

\begin{tabular}{|c|c|c|c|c|c|c|c|c|c|c|c|c|c|c|}
\hline \multirow{3}{*}{ No } & \multirow{3}{*}{ Items } & \multicolumn{10}{|c|}{ Alternatives } & \multirow{3}{*}{$\mathrm{N}$} & \multirow{3}{*}{$\begin{array}{l}\text { Total } \\
\text { Score }\end{array}$} & \multirow{3}{*}{$\begin{array}{l}\text { Average Score Per } \\
\text { Item }\end{array}$} \\
\hline & & \multicolumn{2}{|l|}{5} & \multicolumn{2}{|l|}{4} & \multicolumn{2}{|l|}{3} & \multicolumn{2}{|l|}{2} & \multicolumn{2}{|l|}{1} & & & \\
\hline & & $\mathrm{F}$ & $\%$ & $\mathrm{~F}$ & $\%$ & $\mathrm{~F}$ & $\%$ & $\mathrm{~F}$ & $\%$ & $\mathrm{~F}$ & $\%$ & & & \\
\hline \multicolumn{15}{|c|}{ Indiator of continuus monitoring } \\
\hline 1 & There is a strategy to evaluate control activities & 17 & 19.54 & 65 & 74.71 & 2 & 2.30 & 3 & 3.45 & 0 & - & 87 & 357 & 4.10 \\
\hline 2 & Accuracy and responsibility on financial statements & 34 & 39.08 & 49 & 56.32 & 2 & 2.30 & 2 & 2.30 & 0 & - & 87 & 376 & 4.32 \\
\hline \multirow[t]{2}{*}{3} & Relevant information is used by the leaders to address the problem & 12 & 13.79 & 71 & 81.61 & 2 & 2.30 & 2 & 2.30 & 0 & - & 87 & 354 & 4.07 \\
\hline & Mean & & 24.14 & & 70.88 & & 2.30 & & 2.68 & & - & & 362.3 & 4.16 \\
\hline \multicolumn{15}{|c|}{ Indicators of separate evaluation } \\
\hline 4 & A separate evaluation team develops an evaluation plan & 11 & 12.64 & 65 & 74.71 & 9 & 10.34 & 2 & 2.30 & 0 & - & 87 & 346 & 3.98 \\
\hline 5 & $\begin{array}{l}\text { A separate evaluation team understands the vision, mission and objectives of } \\
\text { government agencies }\end{array}$ & 15 & 17.24 & 64 & 73.56 & 6 & 6.90 & 2 & 2.30 & & - & 87 & 353 & 4.06 \\
\hline \multirow[t]{2}{*}{6} & Weaknesses found immediately communicated & 26 & 29.89 & 58 & 66.67 & 3 & 3.45 & & - & 0 & - & 87 & 371 & 4.26 \\
\hline & Mean & & 19.92 & & 71.65 & & 6.90 & & 1.53 & & - & & 356.67 & 4.10 \\
\hline \multicolumn{15}{|c|}{ Indicators of completion of the audit } \\
\hline 7 & Review and evaluate audit results & 34 & 39.08 & 53 & 60.92 & & - & & - & & - & 87 & 382 & 4.39 \\
\hline 8 & Leaders and auditors monitor audit results & 24 & 27.59 & 63 & 72.41 & & - & & - & & - & 87 & 372 & 4.28 \\
\hline \multirow[t]{2}{*}{9} & Certain transaction or event issues are corrected immediately & 35 & 40.23 & 52 & 59.77 & & - & & - & & - & 87 & 383 & 4.40 \\
\hline & Mean & & 35.63 & & 64.37 & & - & & - & & - & & 379.00 & 4.36 \\
\hline \multicolumn{3}{|c|}{ Mean on the Variable of Control Activities } & 26.56 & & 68.97 & & 3.07 & & 1.40 & & 0.00 & & 366.00 & 4.21 \\
\hline
\end{tabular}

Source: Primary Data Analysed, 2016. 
Table 6 - Frequency Distribution Respondents' Answers on Fraud

\begin{tabular}{|c|c|c|c|c|c|c|c|c|c|c|c|c|c|c|}
\hline \multirow{3}{*}{ No } & \multirow{3}{*}{ Items } & \multicolumn{10}{|c|}{ Alternatives } & \multirow{3}{*}{$\mathrm{N}$} & \multirow{3}{*}{ Total Score } & \multirow{3}{*}{$\begin{array}{l}\text { Average Score } \\
\text { Per Item }\end{array}$} \\
\hline & & \multicolumn{2}{|r|}{5} & \multicolumn{2}{|r|}{4} & \multicolumn{2}{|c|}{3} & \multicolumn{2}{|r|}{2} & \multicolumn{2}{|r|}{1} & & & \\
\hline & & $\mathrm{F}$ & $\%$ & $\mathrm{~F}$ & $\%$ & $\mathrm{~F}$ & $\%$ & $\mathrm{~F}$ & $\%$ & $\mathrm{~F}$ & $\%$ & & & \\
\hline \multicolumn{15}{|c|}{ Indicators of making effective control activities } \\
\hline 1 & Review on employee performance & 17 & 19.54 & 59 & 67.82 & 9 & 10.34 & 1 & 1.15 & 1 & 1.15 & 87 & 351 & 4.03 \\
\hline 2 & Review on the achievement of budget realization & 23 & 26.44 & 60 & 68.97 & 2 & 2.30 & 1 & 1.15 & 1 & 1.15 & 87 & 364 & 4.18 \\
\hline 3 & Control over application used & 16 & 18.39 & 66 & 75.86 & 4 & 4.60 & 0 & - & 1 & 1.15 & 87 & 357 & 4.10 \\
\hline 4 & Physical security of assets & 15 & 17.24 & 65 & 74.71 & 5 & 5.75 & 1 & 1.15 & 1 & 1.15 & 87 & 353 & 4.06 \\
\hline 5 & Imposition of job responsibilities & 6 & 6.90 & 44 & 50.57 & 25 & 28.74 & 10 & 11.49 & 2 & 2.30 & 87 & 303 & 3.48 \\
\hline \multicolumn{3}{|c|}{ Mean } & 17.70 & & 67.59 & & 10.34 & & 2.99 & & 1.38 & & 345.6 & 3.97 \\
\hline \multicolumn{15}{|c|}{ Indicators of improving organizational culture } \\
\hline 6 & Supervision to the party performing transactions & 28 & 32.18 & 56 & 64.37 & 1 & 1.15 & 2 & 2.30 & 0 & - & 87 & 371 & 4.26 \\
\hline 7 & Openness in the decision-making process & 32 & 36.78 & 49 & 56.32 & 5 & 5.75 & 1 & 1.15 & & - & 87 & 373 & 4.29 \\
\hline 8 & Effective monitoring on the performance accountability & 26 & 29.89 & 58 & 66.67 & 3 & 3.45 & 0 & - & 0 & - & 87 & 371 & 4.26 \\
\hline 9 & Compliance with applicable laws and regulations & 35 & 40.23 & 50 & 57.47 & 2 & 2.30 & 0 & - & 0 & - & 87 & 381 & 4.38 \\
\hline 10 & Uphold morality, honesty and individual responsibility & 46 & 52.87 & 37 & 42.53 & 4 & 4.60 & & - & 0 & - & 87 & 390 & 4.48 \\
\hline 11 & Competence and professionalism & 49 & 56.32 & 35 & 40.23 & 1 & 1.15 & 2 & 2.30 & 0 & - & 87 & 392 & 4.51 \\
\hline 12 & Commitment to always improve performance & 51 & 58.62 & 33 & 37.93 & 1 & 1.15 & 1 & 1.15 & 1 & 1.15 & 87 & 393 & 4.52 \\
\hline \multicolumn{3}{|c|}{ Mean } & 43.84 & & 52.22 & & 2.79 & & 0.99 & & 0.16 & & 381.57 & 4.39 \\
\hline \multicolumn{15}{|c|}{ Indicators of effective internal audit functions } \\
\hline 13 & Implementation of the audit & 37 & 42.53 & 49 & 56.32 & 1 & 1.15 & & - & & - & 87 & 384 & 4.41 \\
\hline 14 & Studying internal audit reports & 26 & 29.89 & 59 & 67.82 & 2 & 2.30 & & - & & - & 87 & 372 & 4.28 \\
\hline 15 & Respond quickly and decisively to improvement suggestions & 30 & 34.48 & 56 & 64.37 & 1 & 1.15 & & - & & - & 87 & 377 & 4.33 \\
\hline 16 & The existence of strict sanctions on fraud & 43 & 49.43 & 39 & 44.83 & 3 & 3.45 & 2 & 2.30 & & - & 87 & 384 & 4.41 \\
\hline 17 & Rewards to outstanding employees & 33 & 37.93 & 49 & 56.32 & 4 & 4.60 & & - & 1 & 1.15 & 87 & 374 & 4.30 \\
\hline 18 & Assistance to employees who get into trouble & 12 & 13.79 & 55 & 63.22 & 15 & 17.24 & 4 & 4.60 & 1 & 1.15 & 87 & 334 & 3.84 \\
\hline 19 & The existence of clear information on categories of gifts, bribery, and official & 20 & 22.99 & 53 & 60.92 & 10 & 11.49 & 1 & 1.15 & 3 & 3.45 & 87 & 347 & 3.99 \\
\hline 20 & The existence of certain sources to detect fraud & 18 & 20.69 & 57 & 65.52 & 8 & 9.20 & 1 & 1.15 & 3 & 3.45 & 87 & 347 & 3.99 \\
\hline 21 & The existence of channels to report the occurrence of fraud & 20 & 22.99 & 60 & 68.97 & 5 & 5.75 & 2 & 2.30 & & - & 87 & 359 & 4.13 \\
\hline \multicolumn{3}{|c|}{ Mean } & 30.52 & & 60.92 & & 6.26 & & 1.28 & & 1.02 & & 364.22 & 4.19 \\
\hline & Mean on the Variable of Control Activities & & 30.69 & & 60.24 & & 6.46 & & 1.75 & & 0.86 & & 363.80 & 4.18 \\
\hline
\end{tabular}

Source: Primary Data Analysed, 2016. 
Table 7 - Recapitulation of Multiple Regression Analysis Result

\begin{tabular}{|l|l|l|l|}
\hline Dependent Variable $(\mathrm{Y})=$ Fraud Prevention & Coefficient of Regression & $\mathrm{t}$ & sig. \\
\hline Variable & 9.723 & 1.826 & 0.071 \\
\hline Constanta & 0.183 & 1.848 & 0.068 \\
\hline Control Environemnt $(\mathrm{X} 1)$ & 0.430 & 2.025 & 0.046 \\
\hline Risk Assessment $(\mathrm{X} 2)$ & 0.030 & 0.307 & 0.760 \\
\hline Control Activities $(\mathrm{X} 3)$ & -0.022 & -0.079 & 0.938 \\
\hline Information and Communication (X4) & 0.928 & 3.553 & 0.001 \\
\hline Monitoring (X5) & $=0.825$ & & \\
\hline $\mathrm{R}$ & $=0.681$ & & \\
R square (R2) & $=$ & & \\
Ajusted R Square & 0.661 & & \\
F Count & $=34.563$ & & \\
Sig F & $=0.000$ & &
\end{tabular}

Source: Regression Results.

This is in accordance with the results of research by Meliany and Hernawati (2013) which state that the effectiveness of internal control significantly influences the tendency of fraud. The leaders of government agencies should ensure that internal control functions are running as expected. To that end, the leaders of government agencies need to perform all aspects or elements in the government's internal control system, i.e. the control environment, risk assessment, control activities, information and communication, and monitoring. Thus, simultaneously internal control influences fraud prevention.

Partial test is intended to see the influence of each independent variable to the dependent variable. The results are as follows. Control environment partially and significantly influences fraud prevention. Risk assessment partially and significantly influences fraud prevention. Control Activities partially and significantly influences fraud prevention. Information and communication partially and significantly influences fraud prevention. Monitoting partially and significantly influences fraud prevention. Control environment, risk assessment, control activities, information and communication, and monitoring have significant effect on fraud prevention lin the local government of Palu.

Furthermore, from the analysis, it can be interpreted that every improvement of control environment, risk assessment, control activities, information and communication, and monitoring will give improvement to fraud prevention of. This is also supported by an interview with Mr. Ayub Sukresno (Accountability Auditor for Local Government) on October 21, 2016 at the office of BPKP (Supervisory Agency for Finance and Development). He stated that the internal control system of the government regulates things thoroughly, but the internal control system of the city government has not been fully applied. This is evidenced by the assessment of BPKP that the city government of Palu is still at level 1 , whereas the target of BPKP itself for a city government is at level 3 for good internal control. Level 1 means that there is infrastructure problem.

The local government has not yet built infrastructure. Infrastructure here refers to the regulation-in which each local government must have rules of ethics or employee ethics. However, so far the local government of Palu only refers to Government Regulation Number 58 , which should have been changed into operational standard of behavior. Most municipal governments in Indonesia still have not implemented this as well. In addition to the existing infrastructure, socialization is still lacking so the application of SPIP is not maximized.

Risk assessment is a management activity supported by employees who understand about risk management activities. Elements of risk assessment have been done well by the government of Palu. This is supported by interview with Mr. Arifin Haerolla, SH, M.Si (an auxiliary inspector of Regional 1) on October 18, 2016 at the inspectorate office of Palu. He declares that fraud can be detected by a good working system and mechanisms, if each employee performs the duties properly. Employees must be able to control and assess the risks of work and control the risks based on the tasks at various stages of work; this way, we know whether the work is done in accordance with the procedures or regulations, and error will be easier to detect as to know fraud. 
Financial reporting in the city government of Palu is still not well managed, as stated by Mr. Arifin Haerolla, SH, M.Si (an auxiliary inspector of Regional 1) on October 18, 2016 at the inspectorate office of Palu; he asserts that this happesn due to the problem of assets. Annual asset purchases increase but year-end financial reporting is often missing, due to lack of good system governing the management of assets, whereas assets are important in the preparation of financial statements.

Elements of monitoring have been done well by the city government of Palu as stated by Mr. Arifin Haerolla, SH, M.Si (an auxiliary inspector of Regional 1) on October 18, 2016 at the inspectorate office of Palu. He confirms that after the examination, the inspectorate always do the controlling steps. As an example, when there are findings on mistakes by certain SKPD, then the inspectorate provides 60 days to correct the mistake, yet, if no feedback from the SKPD concerned during the 60 days given, the the inspectorate will come again to remind. Usually, most SKPDs directly make improvements.

Monitoring is required by the leaders as it is a tool to assure the effectiveness of the internal control system. This way, they can continuous its implementation if it is proven to be effective, and at the same time, they can identify changes that occur. Implementation of monitoring on the city government of Palu has an effect on fraud prevention.

\section{CONCLUSION}

Based on the afore-mentioned results, the following conclusions can now be drawn related to fraud prevention in the city government of Palu, as follows:

Control Environment, Risk Assessment, Control Activities, Information and Communication, and Monitoring show overall significant effect toward fraud prevention in the local government of Palu;

Control Environment has no significant effect toward fraud prevention in the local government of Palu. Risk Assessment has significant effect toward fraud prevention in the local government of Palu. Control Activities have no significant effect toward fraud prevention in the local government of Palu. Information and Communication has no significant effect toward fraud prevention in the local government of Palu. Monitoring has significant effect toward fraud prevention in the local government of Palu.

\section{REFERENCES}

1. Agoes, S. (2012). Auditing. 4th Edition. Salemba Empat, Jakarta.

2. Comitte of sponsoring organizations of the treadway commission. 2013. Internal control integrated framework. Retrieved from www.coso.org on January 18, 2015.

3. Ikatan Akuntan Indonesia Per 1 September 2007. Standar Akuntansi Keuangan. Jakarta: Salemba Empat.

4. Meliany, L. and Hernawati, E. (2013). Pengaruh Keefektifan Pengendalian Internal Dan Kesesuaian Kompensasi Terhadap Kecenderungan Kecurangan Akuntansi. Sustainable Competitive Advantage (SCA), Vol. 3 No. 1.

5. Purba, B. (2015). Fraud dan Korupsi. Lestari Kiranatama, Jakarta.

6. Pusat Pendidikan dan Pelatihan Pengawasan Badan Pengawasan Keuangan dan Pembangunan. (2008). Sistem Pengendalian Intern Pemerintah. Pusdiklatwas BPKP, Bogor. 